\title{
Los sistemas defensivos del Real Arsenal de Cartagena (S. XVIII) ${ }^{1}$ José Antonio Martínez López \\ Universidad Católica San Antonio Murcia. Murcia, España, jalopez712@ucam.edu
}

\begin{abstract}
Cartagena and its arsenal became the main facility of the Spanish Mediterranean during the 18th century. Dozens of fortification were built in order to defend them, which were organised into three large systems of defence. The first was created for the immediate protection of the shipyard by means of perimeter ramparts, which enclosed the facilities, and a battery to the port side. The second was associated with marine defence and was formed by a series of defence batteries built at the mouth of the harbour. Finally, land defence was dealt with by means of the construction of town ramparts and several external forts on the main hills. These defences managed to protect the arsenal and the city against possible attacks from outside and the majority remained in service until the beginning of the 20th century, when a large number were abandoned.
\end{abstract}

Keywords: Cartagena, Real Arsenal, defense system.

\section{Introducción}

Al finalizar la Guerra de Sucesión, con el cambio dinástico España inició un periodo de profundas reformas que se generalizaron a lo largo del siglo XVIII. Fueron de especial trascendencia las aplicadas en el ámbito naval en su más amplio sentido (Pérez, 1980).

El dominio del mar fue una cuestión estratégica ya que para poder conservar los territorios de ultramar y garantizar el comercio hubo que contar con los medios marítimos adecuados. Por ello las naciones europeas edificaron en sus principales puertos una serie de bases navales con el objeto de poder construir las flotas y mantenerlas. Para la defensa de los arsenales se desarrollaron ingentes obras de fortificación.

En España, bajo el mandato del ministro Patiño se planificó una profunda renovación de la Armada con la creación de una nueva estructura organizada en Departamentos Marítimos cada uno de ellos asociado a un arsenal: Norte, Mediodía y Levante, este último con sede en Cartagena (Pérez, 2005). En estos arsenales se construyeron decenas de navíos tecnológicamente muy avanzados, permitiendo que España fuese en el siglo XVIII una potencia naval de primer orden.

El Arsenal de Cartagena fue la culminación de un largo proyecto que perseguía dotar a la ciudad de unas infraestructuras portuarias y que se inició hacia 1540 con la designación de la ciudad como una de las sedes de la Proveeduría de Armadas y Fronteras, institución dedicada, entre otras funciones, al mantenimiento y avituallamiento de las flotas de galeras, labor centralizada en la Casa del Rey. Este proyecto continuó en 1670 con el plan que Lorenzo Possi propuso para construir un puerto donde pudiesen fondear las flotas al haber sido designada Cartagena dos años antes base permanente de las Galeras de España. Hubo que esperar a 1749 para la aprobación e inicio de los trabajos de un proyecto más ambicioso, el de un arsenal con su marina completa para construir y mantener los navíos de la Real Armada y cuyas obras se 
prolongaron hasta 1782. Una parte de ese arsenal continua hoy día cumpliendo las funciones para las que fue diseñado (Peñalver, 2011) ${ }^{2}$.

A lo largo del siglo XVIII el Arsenal de Cartagena se convirtió en la instalación industrial más importante del Mediterráneo español, generando un significativo aumento demográfico y transformando la trama urbana con la proyección y ejecución de numerosas obras civiles y militares.

A lo largo de los últimos años están viendo la luz bastantes trabajos de investigación sobre diversos aspectos relacionados con esta instalación (Anca, 2007), (Iniesta, 2002), (Melendreras, 2009), (Rubio, 1988), (Rubio, 2001), (Roda, 2003).

Este artículo aborda de forma sintética los tres sistemas defensivos que se crearon para la protección del arsenal. El primero de ellos fue el desarrollado para la propia seguridad del astillero, formado por un muro perimetral y una batería en el frente portuario; el segundo se desplegó en el frente marítimo y estuvo compuesto por una serie de baterías costeras; y por último, el sistema defensivo terrestre formado por una fortificación urbana abaluartada y una serie de fuertes exteriores situados sobre las principales cumbres que dominan la población.

\section{La defensa inmediata del arsenal. El muro perimetral y la batería.}

\subsection{El muro perimetral}

La preocupación por la seguridad del arsenal fue una cuestión prioritaria desde el primer momento ya que había que controlar el acceso de personas al astillero e impedir la sustracción de materiales, y al mismo tiempo evitar sabotajes que pudiese arruinar todo lo que se estaba edificando. Por ello se tomaron medidas extraordinarias y se dictaron normas tan estrictas como por ejemplo prohibir la entrada al recinto con fósforos. No hay que olvidar que las materias primas con las que se trabajaba en su interior eran altamente inflamables: maderas, cáñamo, algodón, betunes, etc.
A finales de 1749, Feringán envió al marqués de la Ensenada el proyecto y el presupuesto de la defensa perimetral para cerrar el arsenal $^{3}$. Propuso cercarlo con un muro de cal y canto de 15 pies de alto coronado de lajas de sillería de tres pies de grueso y además planteó que entre el arsenal y la ciudad quedase una calle de 26 tuesas de ancho (Pérez-Crespo, 1992).

Este muro se inició en las proximidades de la puerta del Muelle para cambiar su trayectoria en el comienzo de la calle Real, espacio nuevo que se generó a partir de la demolición de una buena parte del caserío de esta zona. Esta calle delimitó en toda su extensión el perímetro del muro por la parte oriental de la ciudad hasta llegar a la rambla de Benipila, donde se unió y aprovechó las tierras del malecón realizado para desviar sus aguas a la Algameca Chica. Hacia esta dirección discurrió un nuevo tramo del muro hasta llegar a las inmediaciones de la falda del monte de las Galeras, donde tras marcar un nuevo giro al este, a través de la parte inferior de su ladera continuó hasta llegar al mar, en el mismo punto donde se encontraba la caseta que guardaba la cadena que diariamente se desplegaba por la noche para cerrar la bocana de la dársena del arsenal.

Las obras del muro dieron comienzo el 9 de septiembre de 1750 tal y como había proyectado Sebastián Feringán y en 1762, año en el que fallece el ingeniero, la cerca estaba finalizada en su totalidad.

\subsection{La batería del Arsenal}

Para la zona marítima del arsenal Feringán proyectó la construcción de un frente de fortificación que protegiese su entrada. Debía ubicarse o bien en el propio arsenal o más adelantado en lo que denominó en su proyecto el muelle para el comercio ${ }^{4}$.

Esta última infraestructura, la más avanzada en el puerto, no se construyó, por lo que la defensa de la fachada marítima del arsenal quedó retranqueada y organizada en un frente de fortificación formado por dos medios baluartes unidos por una cortina, un hornabeque.

El medio baluarte situado al oeste se denominó de Santa Bárbara y el situado en el extremo 
opuesto, que en principio se proyectó como un baluarte completo, cuyo flanco debía aprovechar el antiguo muelle y unirse con la nueva muralla de la ciudad que se había proyectado, se denominó baluarte de San Fernando.

Su construcción fue compleja ya que la obra tuvo que cimentarse sobre lodos y fue necesario utilizar un sistema de pilotaje hincando decenas de troncos de pino para poder asentar sobre ellos los grandes sillares de piedra marmoleña de los alzados. Además, esta estructura sirvió por su cara interna para contener los rellenos que se estaban depositando para formar una gran explanada sobre la que se edificaron diversas instalaciones del arsenal, destacando el Cuartel de Presidiarios y Esclavos.

Este frente defensivo se conoció como batería del Arsenal y fue artillado con cerca de 40 piezas, convirtiéndose en el último reducto del complejo sistema defensivo frente a un ataque marítimo. De tal forma que un navío enemigo que pretendiese atacar el arsenal necesitaría contar con viento favorable, sortear las diversas lajas de la bocana y al mismo tiempo evitar no quedar desarbolado por el fuego masivo de las baterías de la entrada del puerto y además evitar la artillería situada en el castillo y en la propia fortificación urbana dispuesta sobre los baluartes marítimos y por último enfrentarse a la batería del Arsenal.

Hoy día tan sólo se conserva la cortina ya que los baluartes de los extremos fueron desmontados en posteriores obras, el de Santa Bárbara en los años 60 del siglo pasado con el objeto de ampliar la bocana del arsenal. Esta batería ha sido recientemente recreada con la colocación por parte del Museo Naval de varias piezas de artillería que rememoran su primitiva función.

\section{La defensa marítima. Las baterías de la bocana del puerto}

En el siglo XVI y de forma progresiva se generalizó el uso de la artillería pirobalística en los navíos aumentando considerablemente su poder destructivo, por ello fue necesario desarrollar sistemas defensivos estáticos capaces de batir con fuego masivo las flotas enemigas.
No fue hasta la mitad del siglo XVII cuando se materializó la construcción de la primera fortificación en la entrada del puerto tras el fracaso de varios intentos previos (Rubio $2000 /$ Velasco, 2009). Hubo que buscar una solución que estratégicamente fuese satisfactoria y económicamente viable. El lugar elegido fue la punta de Trincabotijas, situada en la falda del monte de San Julián. Allí se realizó el 9 de septiembre de 1640 un ensayo artillero con el objeto de comprobar si desde aquí los fuegos podían cubrir tanto la entrada del puerto como Escombreras y las Algamecas. Varios disparos dejaron de manifiesto el éxito del ensayo dando comienzo las obras de una nueva fortificación en este enclave, la primera del futuro sistema defensivo del puerto de Cartagena.

El 10 de mayo de 1683, ante la ruptura de hostilidades con Francia y la presencia de su flota en el Mediterráneo, se ordenó la construcción de nuevas fortificaciones en las puntas de poniente con el objeto de cerrar la bocana con fuego cruzado, en concreto se eligieron las puntas de Podadera y Navidad ${ }^{5}$. La elección de estos puntos geográficos fue una solución muy eficaz, pues no sólo se controlaba el acceso al puerto, sino también a las Algamecas y Escombreras.

Al final de la Guerra de Sucesión estas defensas costeras estaban en un estado de ruina total ${ }^{6}$. Esta situación se mantuvo hasta la segunda mitad del siglo XVIII cuando, paralelamente a la construcción del arsenal, se diseñó un sistema defensivo permanente.

El ingeniero Esteban Panón fue destinado a Cartagena con esta misión y desde septiembre de 1739 a los primeros meses de 1740 se encargó de proponer el plan de defensa general del puerto de Cartagena que fue inmediatamente aprobado dando a continuación inicio las obras ${ }^{7}$. En una primera fase los trabajos se centraron en poner en estado de defensa las baterías ya existentes: Trincabotijas, Navidad, Santa Ana y Podaderas; y en proyectar y ejecutar una serie de baterías a barbeta como la de Santa Florentina, San Isidoro, San Leandro o Algameca Chica. Tras finalizar la primera fase abordó la 
construcción de una segunda batería en la Podadera, otra en Escombreras, la defensa del monte de las Galeras y dos baterías dentro de la dársena: San Nicolás y San Alejo. A principios de 1743, Panón finalizó su estancia en Cartagena, en tres años había conseguido dejar formado el sistema defensivo de la bahía de Cartagena.

Quedó configurado en el frente de levante por cuatro fortificaciones. En San Leandro una batería articulada en tres niveles. Siguiendo la línea de costa San Isidoro y Santa Florentina, comunicadas entre sí y que por su emplazamiento cubrieron también la entrada a las Algamecas. A poca distancia se dispuso la batería de Santa Ana. Finalmente, para cerrar la entrada a Escombreras se construyeron las baterías de Trincabotijas, Alta y Baja. En el frente de poniente, la batería de la Podadera (llamada también de San Juan) quedó establecida en dos reductos: Podadera Baja que protegió el puerto y Alta que defendió las Algamecas. La batería de Navidad cruzó sus fuegos con la de Santa Ana y cubrió la entrada a la bahía de Escombreras. Igualmente se estableció en el Espalmador una batería, la del Apostolado. Finalmente, al pie del castillo de la Concepción se levantaron de nuevo dos baterías: San Nicolás y San Alejo y se remozó la de San Carlos. En Escombreras, el ingeniero propuso levantar una batería o fuerte en la Uña del Gato, próxima a Trincabotijas, para defender directamente la entrada a esta bahía por la parte izquierda.

A lo largo de los años siguientes sucesivos ingenieros acometieron actuaciones de mantenimiento ${ }^{8}$ y algunas obras nuevas ante el estado de guerra con otros países, como es el caso de las baterías provisionales construidas en 1770 en el Soto de la Podadera con tierra y fajina con motivo de la ruptura de hostilidades con Inglaterra ${ }^{9}$. De todos estos trabajos queda una documentación exhaustiva que permite seguir de forma detallada la evolución de este sistema defensivo y sus baterías, que en su mayor parte se mantuvieron operativas hasta principios del siglo XX.

\section{La defensa terrestre. La fortificación urbana y los fuertes exteriores}

Fortificada la bocana, continuó existiendo una cuestión de trascendental importancia, la defensa del arsenal y la ciudad por el frente de tierra. Estando bien avanzadas las obras del arsenal, las defensas urbanas existentes, las construidas bajo el reinado de Felipe II en el siglo XVI, se encontraban en un estado lamentable, prácticamente arruinadas en su totalidad y además no protegían al arsenal ya que el lugar donde se estaba edificando se encontraba fuera del perímetro amurallado (Martínez, 2014). Esta situación se mantuvo hasta que se ejecutó el proyecto de defensa terrestre, consistente en una muralla abaluartada y en una serie de fuertes exteriores sobre las principales alturas que dominaban la ciudad.

Estas fortificaciones fueron el resultado de un largo proceso con innumerables reflexiones realizadas por diversos ingenieros que quedaron plasmadas en las memorias y proyectos redactados a lo largo de casi un siglo (Rubio, 1991).

A partir de la segunda mitad del siglo XVII, cuando se decidió que Cartagena fuese la base de las Galeras y Navíos de S.M., los primeros ingenieros que llegaron para proyectar la adecuación del puerto trabajaron paralelamente en una propuesta para mejorar la fortificación de la ciudad (Munuera, 2003).

En 1725, Antonio Montaigut de la Perille, tras un análisis del estado de las defensas, planteó en su proyecto y presupuesto una serie de propuestas para las fortificaciones de la ciudad. Dicho proyecto fue pronto juzgado por el también ingeniero Alejandro de Rez, que llegó a Cartagena en 1728, cuando ya se había creado el Departamento Marítimo del Mediterráneo ${ }^{10}$.

El 5 de mayo de 1732, falleció el ingeniero Alejandro de Rez, siendo sustituido como ingeniero director por Sebastián Feringán, que en 1747 elaboró un proyecto global para la fortificación y defensa de plaza y arsenal con líneas delimitadas que separaban uno y otro ${ }^{11}$.

En 1765, el conde de Aranda, capitán general de los reinos de Valencia y Murcia, permaneció en 
la ciudad durante dos meses, dedicándose a estudiar sus defensas. En un informe señaló que la plaza estaba totalmente indefensa y el arsenal expuesto a un golpe de mano. Inmediatamente se comisionó al ingeniero Pedro Martín Zermeño para proyectar la fortificación. El 9 de mayo de 1766, Zermeño remitió una carta al conde de Aranda en la que le indicó que había terminado el proyecto acompañado de siete planos ${ }^{12}$.

El 17 de noviembre de 1767, llegó a Cartagena el nuevo gobernador, el mariscal de campo Miguel de Irumberri y Balanza, quien tras ver la dificultad de poder ejecutar el proyecto de Zermeño planteó una nueva propuesta de fortificación acompañada de un plano, que se aprobó por Real Orden de 15 de julio de 1768.

Trascurrieron dos años y de nuevo nada se había ejecutado, por lo que se decidió comisionar a otro ingeniero, Francisco Llobet quien estuvo varios meses trabajando en la redacción de un nuevo proyecto. Con fecha 2 de junio de 1770 , expuso sus propuestas en una memoria acompañada de un plano general y una colección de varios planos de detalle en los que se recogieron diversas secciones y plantas ${ }^{13}$.

El proyecto de Llobet fue remitido a Pedro Martín Zermeño quien tras su examen realizó un informe fechado el 25 de agosto de 1770 al que tituló Adiciones al proyecto de Llobet.

Con el proyecto de Francisco Llobet junto con las indicaciones de Silvestre Abarca y con las adiciones de Pedro Martín Zermeño, el ingeniero general Juan Martín Zermeño firmó un plano con fecha 25 de agosto de 1770, en cuya descripción y leyenda quedó plasmado lo que definitivamente se debía ejecutar ${ }^{14}$.

Las obras dieron comienzo y en la planificación de las tareas se planteó iniciar los trabajo de la muralla y posteriormente los fuertes exteriores. Sin embargo, en noviembre de 1772 se produjo un cambio de criterio y se paralizaron los trabajos de la muralla y se abordó la construcción de tres de los fuertes exteriores: Galeras, Atalaya y Moros.

La dirección de las obras iniciales estuvo a cargo de Llobet y no exenta de problemas por los cambios tan sustanciales que éste realizó sobre el proyecto aprobado por lo que tuvo serias discrepancias con Mateo Vodopich y con Juan Martín Zermeño.

Llobet cesó el 14 de febrero de 1773 y fue sustituido por Vodopich. En 1778 finalizaron las obras de los fuertes de Galeras, Atalaya y Moros y se reanudó la construcción de lo que quedaba pendiente de la muralla y los baluartes, tareas que se prolongaron hasta $1788^{15}$.

La fortificación terrestre cubrió en su totalidad los frentes del Mar, Batel, Tierra, Benipila y Galeras. La construcción tuvo un desarrollo lineal de varios kilómetros de extensión con una sucesión de baluartes, 18 en total, unidos por cortinas y cuyos únicos huecos fueron las puertas y poternas.

\section{Conclusiones}

Al finalizar el siglo XVIII Cartagena y su arsenal disponían de unos sistemas defensivos plenamente operativos y capaces de disuadir cualquier acción ofensiva, como se puso de manifiesto durante la Guerra de Independencia, cuando a lo largo de todos los años de contienda las tropas napoleónicas optaron por no atacar la ciudad y ni tan siquiera se plantearon la posibilidad de proceder a su asedio.

A lo largo del siglo XIX las defensas que formaron parte de estos sistemas fueron sufriendo continuas transformaciones para poder adecuarse a los modernos métodos de fortificación y a las nuevas piezas de artillería de retrocarga (Martínez, 2009)

A principios del siglo $\mathrm{XX}$ las fortificaciones terrestres fueron abandonadas por obsoletas e inoperativas. Una buena parte del frente de tierra de la muralla fue demolido, junto con las tres puertas monumentales de la ciudad. Los fuertes exteriores que completaban la defensa terrestre fueron desartillados y la protección se centró en el sistema defensivo del frente marítimo, que fue ampliado considerablemente con otros nuevos emplazamientos que abarcaron un perímetro de tiro mucho mayor en respuesta a los alcances de los montajes artilleros de los buques acorazados.

La entrada de España en la Organización del Tratado del Atlántico Norte (OTAN) supuso un 
cambio sustancial en la estructura militar que se tradujo en diversos planes para modernizar sus infraestructuras defensivas. Uno de ellos, el Plan Norte, supuso la desaparición definitiva de la mayor parte de las unidades desplegadas en Cartagena y el abandono en su totalidad de lo que pervivía operativo de los sistemas defensivos, en su mayor parte baterías de costa.

Perdido su carácter militar estas fortificaciones y sus edificaciones auxiliares, cambiaron su función y su papel pasando a convertirse en poderosos símbolos culturales con un gran potencial para ser gestionados de muy diversas formas.

En los últimos años se han realizado numerosas actuaciones que han permitido que estos elementos ayuden a ampliar los recursos del territorio potenciando el desarrollo local mediante actuaciones en ámbitos tan diversos como el educativo, con la rehabilitación del Cuartel de Antiguones, el Real Hospital y el Cuartel de Presidiarios y Esclavos para sedes universitarias. En el ámbito cultural y turístico, la rehabilitación del fuerte de Navidad por parte del Consorcio Cartagena Puerto de Culturas, ha permitido disponer de la primera batería de costa recuperada íntegramente y visitable. La gestión medioambiental es otro de los puntos fuertes de las actuaciones que se están llevando a cabo por parte del Ministerio de Medio Ambiente que ha adquirido una serie de baterías costeras con el fin de garantizar la conservación de estos lugares y sus entornos ya que se construyeron en puntos estratégicos con altos valores paisajísticos. Por último, es de destacar la Ruta de las Fortalezas, iniciativa que desde hace años viene desarrollando el Ministerio de Defensa a través de la Armada. Esta prueba deportiva desarrollada alrededor de este patrimonio fortificado permite a miles de ciudadanos conocer y disfrutar cada año de los valores históricos, arquitectónicos, medioambientales, etc., que estas fortificaciones conservan hoy día.

\section{Notas}

Archivo General Militar (A.G.M.)

Archivo General de Simancas (A.G.S)
Archivo Municipal de Cartagena (A.M.C.)

Cartoteca del Centro Geográfico del Ejército (C.C.G.E.)

Museo Naval (M.N.)

(1) Este trabajo se enmarca en el proyecto de investigación "Cartagena y Fortificación" (Código TC/04/13) dentro de los trabajos desarrollados por el Aula de Arquitectura Militar adscrita al Grupo de Investigación Arquitectura, Construcción y Territorio de la Universidad Católica San Antonio.

(2) Plano, del proiecto que se propone para construir un arzenal en el puerto de Cartagena con dársena i los edificios correspondientes al armamento y desarmo de navios de S.M. Fecha 9 de septiembre de 1749. A.G.S., MPD, IV-76.

(3) Plano, perfil y elevaciones con que se a de construir el muro para cerrar el Arzenal, dibidido de la ciudad por la calle Real que media según el proiecto general. Fecha 9 de septiembre de 1749. A.G.S., MPD, VI-83.

(4) Plano, de parte del proiecto del Arzenal de Cartagena en que se demuestra la corderia, almazen de jarcia nueba, embocadura de la dársena, frente de fortificación $i$ puerto proyectado para el comercio con muelle de escollera. Fecha 7 de septiembre de 1757. A.G.S., MPD, XIII-72.

(5) A.M.C., Acta capitular de 09-09-1640, pp. 324-325.

(6) Fecha: 1700-07-28. A.G.M, Segovia. Sección. $3^{\text {a }}$, división. $3^{\mathrm{a}}$, 78 .

(7) Plano del puerto y bahía de Cartagena demostrando las baterías de cañones y morteros para su resguardo. Fecha 1739-10-31. A.G.S., MPD, XXVII-18.

(8) Plano en que se demuestran las defensas que se han puesto en el puerto de Cartagena, comunicaciones $i$ caminos que se han hecho, de unas a otras para su fácil manejo i socorros de que carecían. Por Sebastián Feringán Cortés. Fecha 1762-04-24. A.G.S., MPD XXVIII- 6.

(9) Plano de la plaza y puerto de Cartagena,..., en el que se demuestra el estado actual de sus defensas, edificios militares y arsenal de 
Marina. Por Mateo Vodopich. Fecha 1772-0523. C.C.G.E. sign. LM- $3^{\mathrm{a}}-2^{\mathrm{a}}-\mathrm{c}-\mathrm{n}^{\mathrm{o}} 13$.

(10) Plano de parte de la ciudad de Cartagena con indicación de los proyectos de fortificación de don Antonio Montaigu y don Alejandro de Rez. Fecha 1732-10-22. A.G.S., MPD, XVI-46.

(11) Plano de la Plaza de Cartagena con parte de su cuertpo i proiecto original que se propone para fortificarla $i$ construir puerto i dársena... Por Sebastián Feringán Cortés. Fecha 1747-0610. M.N. Cartagena.

(12) Plano de la Plaza de Cartagena, su Arsenal, Puerto y Baterías que le defienden, con el Proyecto de fortificación que de orden de S.M. se propone. Por Pedro Martín Zermeño. Fecha 1766-04-30. M.N. Madrid sign. XLIII-10.

(13) Plano de la plaza de Cartagena con su arsenal y terrenos inmediatos, en que se haze demostración del proyecto de un recinto para ponerla en estado de poderse defender de un golpe de mano, conforme S.M. tiene resuelto... Por Francisco Llobet. Fecha 1770-06-02. C.C.G.E., LM-3 $3^{\mathrm{a}}-2^{\mathrm{a}}-\mathrm{c}-\mathrm{n}^{\mathrm{o}} 11$ (94).

(14) Plano de la plaza de Cartagena en que se propone cerrarla con un de vil recinto, adaptándole defensas proporcionadas a libertar su arcenal de un golpe de mano que pudieran intentar los enemigos. Por Juan Martín Zermeño. Fecha 1770-08-25. C.C.G.E. LM - 3.a $-2 . \mathrm{a}-\mathrm{c}-\mathrm{n}^{\mathrm{o}} 10(100)$.

(15) Plano de la línea magistral del recinto de la Plaza de Cartagena y del fuerte del monte de Galeras...; igualmente se manifiesta en los perfiles que pasan por cada cortina, la altura y disposición en que se halla dicha línea magistral. Por Mateo Vodopich. Fecha 1780-1201. A.G.S., MPD, LI-30.

\section{Referencias}

Anca Alamillo, A. (2007), El arsenal de Cartagena en el siglo XIX:(descripción de las obras, dependencias, talleres y construcción naval durante el periodo de 1801 a 1908), Fundación Alvargonzález, Ed. Gijón, p. 133.

Iniesta A., Martínez J.A. (2002), Estudio y catalogación de las defensas de Cartagena y su bahía, Dirección General de Cultura Ed., Murcia, p.689.

Martínez J.A. (2009), Álbum fotográfico de los fuertes y baterías del puerto de Cartagena (1901), Cartagena Puerto de Culturas Ed. Cartagena, p.68.

Martínez J.A., Nogera J.M., Madrid M.J., Martínez I., (2014), "Las defensas de la Cartagena renacentista: evidencias arqueológicas recientes de las murallas de Carlos I y Felipe II", Anales de Prehistoria y Arqueología, vol. 30, Universidad de Murcia Ed., Murcia, pp. 179-204.

Melendreras M.C. (2009), La fortificación de la Base Naval de Cartagena en el siglo XVIII: proyectos, mapas y planos, Universidad de Murcia Ed., Murcia, p. 201.

Munuera, D. (2003), “Aproximación al estudio de unas murallas casi olvidadas: el informe del ingeniero militar Lorenzo Possi sobre las fortificaciones urbanas de Cartagena (1669)", Revista Arqueomurcia, $\mathrm{n}^{\circ}$ 1, URL: www.arqueomurcia.com, p.15.

Peñalver, M.J. (2011), La génesis de la dársena del puerto de Cartagena a lo largo del siglo XVIII, Tesis Doctoral, Universidad Politécnica de Cartagena, Cartagena.

Pérez P. E. (1980), "La Marina de Guerra española en los comienzos del siglo XVIII (1700-1718)", Revista General de Marina, tomo 199, Madrid, pp. 137 -155.

Pérez C. (2006), Patiño y las Reformas de la Administración en el reinado de Felipe V, Ministerio de Defensa Ed, Madrid.

Pérez-Crespo M. T. (1992), El Arsenal de Cartagena en el siglo XVIII, Ministerio de Defensa Ed., Madrid.

Roda A. (2003), La modernización del Arsenal de Cartagena durante el gobierno de la Unión Liberal (1858-1863), Universidad de Murcia, Servicio de Publicaciones, Murcia.

Rubio J.M., Piñera A. (1988), Los ingenieros militares en la construcción de la base naval de Cartagena, (siglo XVIII), Servicio de Publicaciones del EME, Madrid, p. 206. 
Rubio J.M. (1991), La muralla de Carlos III en Cartagena, Real Academia Alfonso X El Sabio Ed. Murcia, p. 326.

Rubio J.M. (2000), "Los primeros proyectos de fortificación de la costa de Cartagena (primera mitad del siglo XVII.)", Castillos de España n $^{\circ} 116$, Madrid, pp. 41-45.

Rubio J.M. (2001) Historia de la muralla de Carlos III en Cartagena, C.A.M. Ed., Cartagena, p. 471.

Velasco F. (2009), "La torre de Navidad: un enigma de la historia de Cartagena al descubierto", Cartagena histórica, 29, Aglaya Ed., Cartagena, pp. 28-42.

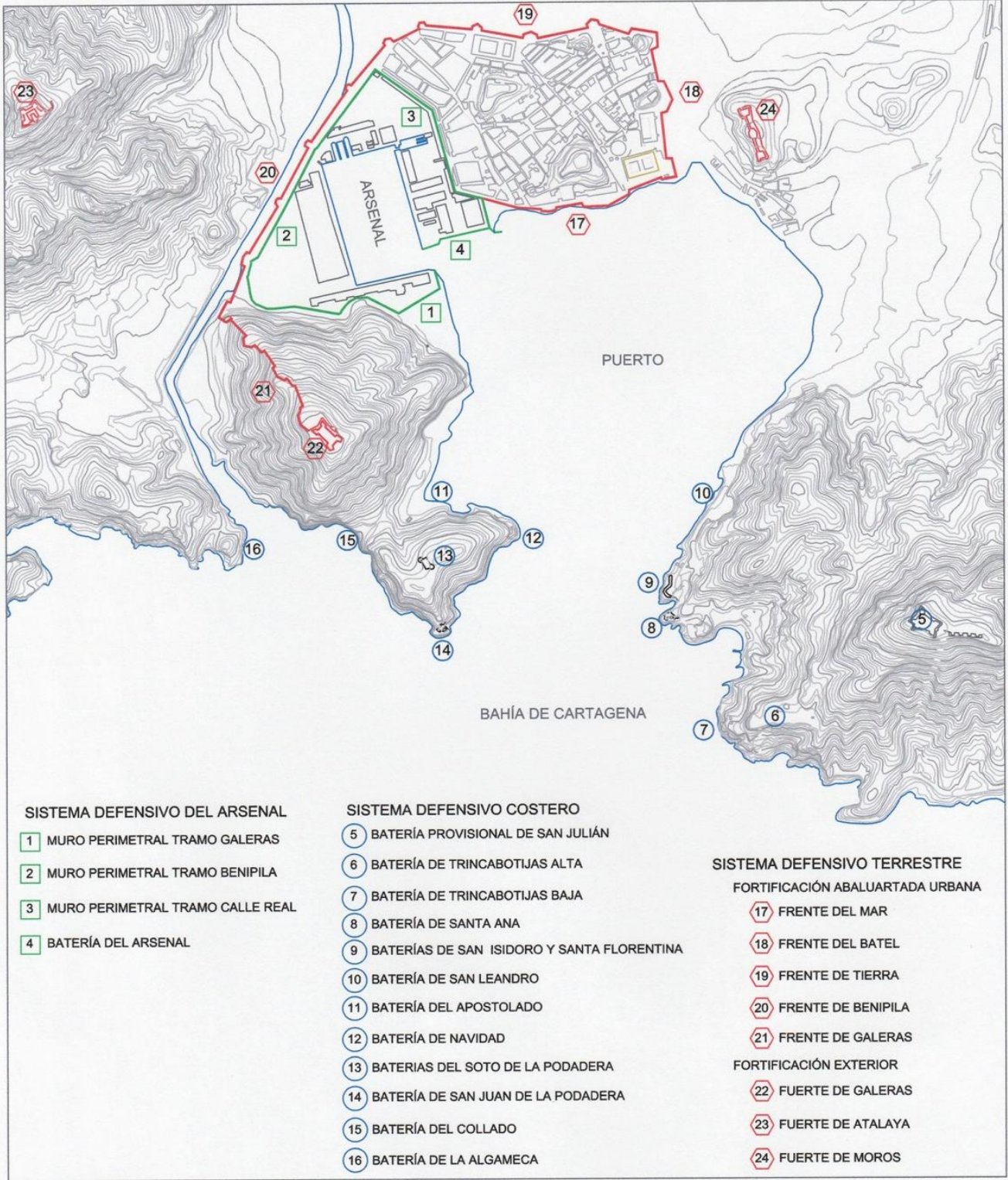

Fig. 1- Los sistemas defensivos del Arsenal de Cartagena y sus fortificaciones 